\title{
Insulin ist notwendig, aber nicht ausreichend: Paradigmenwechsel in der Behandlung des Typ-1-Diabetes
}

\author{
Sandra Lord Carla J. Greenbaum \\ Benaroya Research Institute at Virginia Mason, Seattle, WA, USA
}

\author{
Schlüsselwörter \\ Typ-1-Diabetes · Immuntherapie · Insel-Autoimmunität . \\ Prävention · Teplizumab · TrialNet
}

\section{Zusammenfassung}

Ungeachtet der eindeutigen Belege dafür, dass Typ-1-Diabetes (T1D) bereits deutlich vor einer Manifestation von Hyperglykämie beginnt, stehen keine krankheitsmodifizierenden Therapien für die Behandlung der Erkrankung im Frühstadium zur Verfügung. Nach den hoch interessanten Ergebnissen der Teplizumab Prevention Studie - der ersten Studie, die nachweisen konnte, dass sich ein manifester T1D durch eine Immuntherapie hinauszögern lässt steigt die Hoffnung wieder, dass T1D in Zukunft vor Entwicklung einer Hyperglykämie behandelt werden kann. Es bedarf eines Paradigmenwechsels, da die Mehrheit der Menschen mit T1D die gly-

kämischen Zielwerte nicht erreicht, die mit einem niedrigeren Risiko für T1D-Komplikationen verbunden sind, und bei denen daher weiterhin die Gefahr von Komplikationen und einer verkürzten Lebenserwartung besteht. Die folgende Übersichtsarbeit beschäftigt sich mit der Geschichte und dem aktuellen Stand der Immuntherapie bei T1D und beleuchtet einige Herausforderungen und Ideen für die Zukunft. Auch wenn diese Anstrengungen weltweit unternommen werden, konzentrieren wir uns hier vor allem auf die 2004 aufgelegten Forschungsaktivitäten des Diabetes TrialNet, eines Konsortiums der US-amerikanischen National Institutes of Health.

\section{Einleitung}

Auch knapp 100 Jahre nach der ersten klinischen Anwendung ist Insulin weiterhin das Mittel der Wahl zur Behandlung des Typ1-Diabetes (T1D). Auch wenn am Beginn des T1D eine asymptomatische, aber nachweisbare Insel-Autoimmunität steht, erfolgt eine Behandlung erst mit Auftreten einer Hyperglykämie. Natürlich gab und gibt es Verbesserungen in der Behandlung des T1D, wie etwa zunehmend physiologische Insuline und neue Methoden der Insulinabgabe, die kontinuierliche Glukosemessung und medizintechnische Regelungssysteme, die so genannte «künstliche Bauchspeicheldrüse». Diese Verbesserungen führten zu einer entsprechenden Senkung der Rate von Kurz- und Langzeit-Kom-

plikationen. Allerdings erreicht nur eine Minderheit der Personen mit T1D den Zielwert für Hämoglobin Alc (ein Bluttestparameter, anhand dessen sich die durchschnittliche glykämische Kontrolle über die vorangegangenen zwei bis drei Monate ermitteln lässt), der mit einem niedrigeren Risiko für Komplikationen einhergeht. Diesen Schluss legten die Daten der Organisation T1D Exchange (https://t1dexchange.org) für 2014 nahe. Diesen zufolge wiesen lediglich 17 bis 23\% der Patienten unter 18 Jahren, $14 \%$ der Patienten im Alter von 18 bis 25 Jahren und 30\% der Patienten im Alter über 25 Jahren einen Alc-Wert auf, wie er in den Leitlinien der American Diabetes Association empfohlen wird [1]. Des Weiteren weisen Daten von T1D Exchange aus den Jahren 2016 bis 2018 darauf hin, dass sich diese statistischen Werte un- 


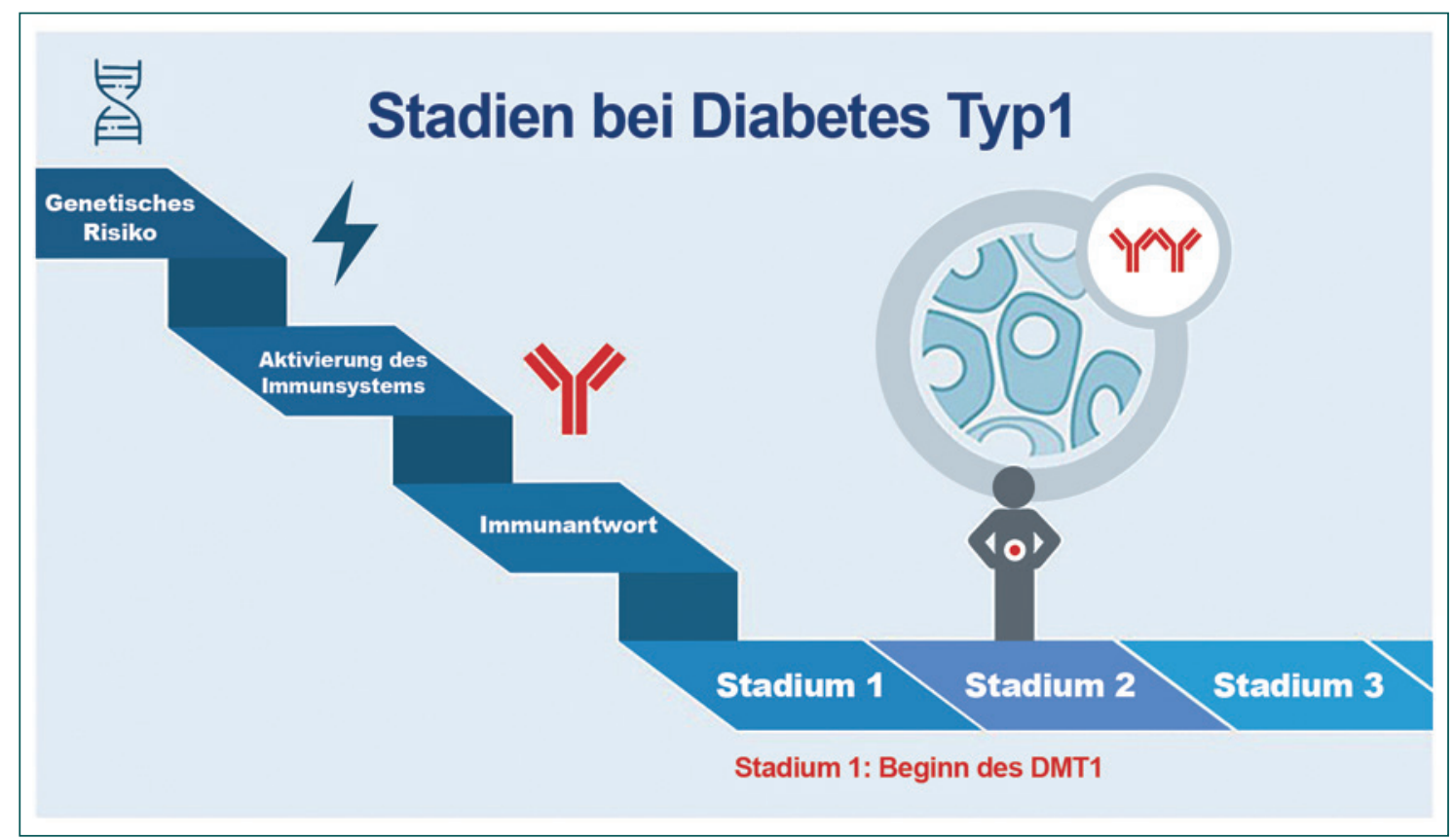

Abb. 1. Die Stadien des Typ-1-Diabetes (T1D). Diabetes TrialNet wurde 2004 von den US-amerikanischen National Institutes of Health mit dem Ziel geschaffen, wissenschaftliche Studien im Bereich der Prävention von T1D durchzuführen. Es handelt sich um ein internationales Netzwerk von Wissenschaftler/inne/n auf dem Gebiet des T1D, die Möglichkeiten der Prävention, Hinauszögerung und Verlangsamung eines Fortschreitens der Erkrankung erforschen. Nachdruck mit Genehmigung des Diabetes TrialNet.

geachtet des verstärkten Einsatzes medizintechnischer Systeme nicht verbessert haben [2]. Selbst bei adäquater glykämischer Kontrolle ist die Behandlung des T1D für Betroffene und ihre Familien mit finanziellen, kognitiven und emotionalen Belastungen verbunden. Es gibt eindeutig einen ungedeckten Bedarf an einer Behandlung des T1D in früheren Stadien der Erkrankung, wenn bereits eine Insel-Autoimmunität vorliegt, aber noch keine Hyperglykämie aufgetreten ist. Ohne Zweifel wird in diesem Zusammenhang die Immuntherapie eine Rolle spielen. Mittlerweile gibt es fünf Immuntherapien, die kurz nach einer T1D-Diagnose die Insulinsekretion nachweislich wirksam aufrecht erhalten. Zudem geben die Ergebnisse der TrialNet Teplizumab Prevention Studie [3] Anlass zu neuem Optimismus, dass ein klinisch manifester T1D hinausgezögert oder sogar gänzlich verhindert werden kann.

\section{Typ-1-Diabetes beginnt mit einer Insel-Autoimmunität}

T1D wurde vor über 40 Jahren erstmals als Autoimmunerkrankung eingestuft, bei der sich in den Langerhans-Inseln der Bauchspeicheldrüse und im Blut inselspezifische Antikörper nachweisen ließen [4, 5]. Seitdem wurde die Krankheitsursache sowohl in Längsschnitt- als auch in Querschnittstudien beschrieben. Hierbei wirken eine erbliche Prädisposition und äußere Auslöser zusammen, auf deren Boden sich auf vorhersehbare Weise eine Insel-Autoimmunität entwickelt. Das höchste genetische Risiko bei Menschen europäischer Abstammung wird durch HLA-Klasse II DR3/4-Gene vermittelt, was auf eine wichtige Rolle von CD4 $4^{+}$-TZellen schließen lässt, auch wenn immer mehr Nicht-HLA-Risi- kogene identifiziert werden [6]. Im Zusammenhang mit T1D gibt es 5 gut validierte Autoantikörper: Antikörper gegen GlutamatDecarboxylase (GAD), Antikörper gegen Insulin (Insulin-Autoantikörper), Antikörper gegen Insulinom-assoziiertes Protein (IA-2), Inselzell-Antikörper und Antikörper gegen einen Zinktransporter (ZnT8). Bei Entwicklung von zwei oder mehr Autoantikörpern ist das Auftreten eines klinisch manifesten T1D unvermeidlich. Dieses im Rahmen einer Konsensus-Konferenz beschriebene Krankheitsmodell [7] wird von Diabetes TrialNet (www.trialnet.org) in Abbildung 1 illustriert. T1D Stadium 1 entspricht hierbei 2 oder mehr Antikörpern mit normaler Glukosetoleranz, Stadium 2 zwei oder mehr Antikörpern mit gestörter Glukosetoleranz, aber ohne persistierende Hyperglykämie, und Stadium 3 einem klinisch diagnostizierten T1D.

Ausgehend davon, dass es sich bei einer Insel-Autoimmunität um T1D im Frühstadium handelt, gewinnt die Identifizierung von Personen mit einem entsprechenden Risiko an Dringlichkeit. Ein Ansatz ist die Untersuchung auf Autoantikörper von Personen mit erhöhtem genetischen Risiko, definiert als das Auftreten von Typ1-Diabetes in der familiären Vorgeschichte oder durch das Testen auf eine Hochrisiko-HLA-Konfiguration bei der Geburt. Allerdings reicht eine Testung allein auf Basis der familiären Vorgeschichte oder des genetischen Risikos nicht aus, um alle Personen zu ermitteln, bei denen die Krankheit auftreten wird, da bei ca. 85\% der von T1D Betroffenen kein Angehöriger ersten Grades an T1D leidet, und lediglich rund 50\% der Menschen europäischer Herkunft mit diagnostiziertem T1D den mit dem höchsten Risiko assoziierten HLA-Klasse II DR3/4-Haplotyp aufweisen. Ein wei- 
Tab. 1. Fünf ausgewählte Immuntherapien mit nachgewiesener Wirksamkeit bezüglich der Aufrechterhaltung der C-Peptid-Konzentration 1 und/oder 2 Jahre nach Randomisierung im Rahmen von Phase-2-Studien zu neu aufgetretenem Typ-1-Diabetes (innerhalb von 100 Tagen nach der Diagnose)

\begin{tabular}{|c|c|c|c|}
\hline Prüfsubstanz & Wirkmechanismus & Verabreichungsart & Referenz(en) \\
\hline Abatacept & $\begin{array}{l}\text { CTLA4-Immunglobulin (lg), } \\
\text { unterbindet die Costimulation: stört } \\
\text { die Antigenpräsentation }\end{array}$ & Monatliche intravenöse (i.v.) Infusion über 2 Jahre & 25,26 \\
\hline Alefacept & $\begin{array}{l}\text { LFA-3-Ig: inhibiert aktivierte T-Zellen } \\
\text { (primär T-Gedächtniszellen) }\end{array}$ & $\begin{array}{l}\text { Zwei Behandlungszyklen mit je } 12 \text { wöchentlichen } \\
\text { intramuskulären (i.m.) Injektionen mit einer 12-wöchigen } \\
\text { Behandlungspause dazwischen }\end{array}$ & 27,28 \\
\hline $\begin{array}{l}\text { Niedrig dosiertes ( } 2,5 \text { mg/kg) } \\
\text { Antithymozytenglobulin (ATG) }\end{array}$ & Lymphozytendepletion & Ein Behandlungszyklus mit 2 i.v. Infusionen über 2 bis 3 Tage & 29,30 \\
\hline Rituximab & $\begin{array}{l}\text { CD20-, B-Zell-Antikörper: stört die } \\
\text { Antigenpräsentation }\end{array}$ & Ein Behandlungszyklus mit 4 wöchentlichen i.v. Infusionen & 31,32 \\
\hline Teplizumab (mehrere Studien & $\begin{array}{l}\text { CD3-Antikörper, inhibiert aktivierte } \\
\text { T-Zellen }\end{array}$ & $\begin{array}{l}\text { Ein Behandlungszyklus mit } 14 \text { täglichen i.v. Infusionen In der } \\
\text { Studie des Tolerance-Network zu Teplizumab bei neu } \\
\text { aufgetretenem T1D (AbATE) wurden nach } 1 \text { Jahr ein zweites } \\
\text { Mal } 14 \text { tägliche Infusionen gegeben. }\end{array}$ & $33,34,35$ \\
\hline
\end{tabular}

terer Ansatz besteht darin, allen Menschen regelmäßige Tests auf Autoantikörper - vielleicht im Vorschulalter - anzubieten. Durch diese Maßnahme ließe sich die Mehrheit der Personen, die bis zur Pubertät T1D entwickeln, identifizieren: In dieser Gruppe lassen sich bereits bei $64 \%$ der 2-Jährigen und bei 95\% der 5-Jährigen Autoantikörper nachweisen [8]. Ein Screening der Allgemeinbevölkerung wurde erstmals in Studie Frlda9 durchgeführt, bei der rund 90000 Kinder aus Bayern im Alter von 2 bis 5 Jahren auf Insel-Autoantikörper getestet wurden. Wie vor kurzem berichtet [9] gelangte man in der Studie Frlda zu dem Schluss, dass das Risiko einer Progression vom T1D-Frühstadium zu klinisch manifestem T1D in der Allgemeinbevölkerung mit demjenigen von Personen mit einer erblichen Vorbelastung für T1D vergleichbar ist. Ebenso wie in anderen Studien stellte man auch im Rahmen von Fr1da fest, dass die Inzidenz einer diabetischen Ketoazidose (DKA) mit 3,2\% zum Zeitpunkt der klinischen T1D-Diagnose niedriger lag als die DKA-Rate zum Zeitpunkt der Diagnose bei herkömmlicher Versorgung, die mit 40 bis 59\% angegeben wird $[10,11]$. Insgesamt stützen die Ergebnisse der Frlda-Studie die Durchführbarkeit und den Nutzen eines bevölkerungsweiten Screenings auf Autoantikörper.

\section{Immuntherapie funktioniert und ist sicher}

\section{Studien bei neu aufgetretenem T1D}

Eine Vielzahl von Immuntherapien wurde bei neu aufgetretenem T1D (innerhalb von 100 Tagen nach Diagnose) daraufhin getestet, inwieweit eine verbliebene endogene Insulinsekretion, gemessen anhand des C-Peptids, aufrecht erhalten werden kann. C-Peptid wird mit Insulin in äquimolaren Mengen sezerniert und kann nach Einleitung einer Insulintherapie als Marker für die endogene Insulinsekretion fungieren. Fünf Immuntherapien mit vertretbarem Nebenwirkungs-/Sicherheitsprofil halten bei neu diagnostiziertem T1D nachweislich die Insulinsekretion aufrecht:
Teplizumab und Otelixizumab [12], Rituximab, Abatacept, niedrig dosiertes Antithymozytenglobulin (ATG) sowie Alefacept. Diese Wirkstoffe und die entsprechenden Studien sind in Tabelle 1 beschrieben. Bemerkenswerterweise zeigt sich die günstige Wirkung der Therapie auf C-Peptid am deutlichsten kurz nach der Randomisierung. Die Abnahme der C-Peptid-Konzentration entspricht letztlich derjenigen in den Kontrollgruppen, was darauf hindeutet, dass die Krankheit durch die Behandlung nicht gestoppt wird. Bei einigen Therapien zeigen sich zwischen den Behandlungsarmen jedoch auch noch nach Jahren signifikante Unterschiede in der C-Peptid-Konzentration. Diese Unterschiede könnten klinisch bedeutsam sein, da die Vorteile einer Aufrechterhaltung der C-Peptid-Konzentration in mehreren Studien nachgewiesen wurden, u. a. ein niedrigeres Risiko für chronische Komplikationen und schwere Hypoglykämie selbst bei Patienten mit kaum nachweisbarer C-Peptid-Konzentration [13-15].

\section{Prävention einer klinisch manifesten Erkrankung}

Auch wenn sich verschiedene Wirkstoffe bei neu aufgetretenem T1D als wirksam erwiesen haben, ist eine Prävention der Erkrankung schwieriger zu erreichen. TrialNet lancierte 2011 die Teplizumab Prevention Studie in einer Kohorte von Hochrisiko-Probanden mit multiplen Autoantikörpern und gestörter Glukosetoleranz, bei denen jedoch kein klinisch manifester T1D vorlag. Teplizumab ist ein monoklonaler CD3-Antikörper, der nicht an den Fc-Rezeptor bindet, sondern die autoreaktive T-Zell-Funktion stört und die Funktion regulatorischer T-Zellen verstärken kann. Die Ergebnisse der TrialNet Studie, im Juni 2019 online im New England Journal of Medicine [3] veröffentlicht, liefern erstmals Belege dafür, dass sich eine klinische Manifestation des T1D durch eine Immuntherapie hinauszögern lässt. Insgesamt 76 Studienteilnehmer erhielten 14 tägliche Infusionen Placebo oder Teplizumab, gefolgt von regelmäßigen Kontrollen in Form von oralen Glukosetoleranztests. Am Ende der Studie wurde bei den Teil- 
nehmern, die Teplizumab erhielten, ein klinisch manifester T1D median 24 Monate später diagnostiziert als bei Probanden unter Placebo [3]. Zwischen den Kohorten unter Placebo und Teplizumab zeigte sich vor allem kein Unterschied in Bezug auf Neuinfektionen, was die Sicherheitsdaten aus den Studien zu Teplizumab bei neu aufgetretenem T1D bestätigt. Eine fortlaufende Beobachtung der Teilnehmer, die bei Studienende noch keinen T1D entwickelt hatten, wird zusätzliche Daten zur Sicherheit, zur möglichen Dauer des Nutzens sowie den Eigenschaften von Probanden, die langfristig auf die Behandlung ansprechen, liefern. Die Teplizumab Prevention Study (Studie zur Prävention mit Teplizumab) bietet eine Grundlage für die Untersuchung weiterer Immuntherapien mit nachgewiesener Wirksamkeit bei neu aufgetretenem T1D vor einer klinischen Manifestation der Erkrankung. TrialNet testet derzeit das CTLA 4-Fc-Fusionsprotein Abatacept bei Personen mit multiplen Autoantikörpern um festzustellen, ob es in der Lage ist, eine klinische Manifestation von T1D hinauszuzögern [16], und wird in Kürze eine Studie mit dem BZell-Antikörper Rituximab mit anschließender Gabe von Abatacept, ebenfalls in einer Risikopopulation, auflegen [17]. Die Studie zu dieser Wirkstoffkombination wird weiter unten (im Abschnitt «Mögliche Synergieeffekte durch eine kombinierte oder sequenzielle Anwendung von Therapien») beschrieben. Eine weitere in Planung befindliche TrialNet Studie soll untersuchen, inwieweit sich die klinische Manifestation eines T1D in einer Hochrisikopopulation mit verschiedenen Autoantikörpern mit Hilfe von niedrig dosiertem ATG hinauszögern lässt.

Während Teplizumab, Abatacept, Rituximab und ATG auf Grundlage der positiven Ergebnisse bei kürzlich diagnostizierten Patienten für eine Anwendung zur Prävention von T1D in Betracht gezogen werden, können andere Therapien im Hinblick auf eine Prävention oder Progression von T1D untersucht werden, ohne zuerst bei neu aufgetretenem T1D getestet worden zu sein. So wurden in früheren Studien parenteral, nasal und oral verabreichtes Insulin [18-21], Nicotinamid [22] sowie eine KuhmilchRezeptur mit hydrolysiertem Casein [23, 24] untersucht. Auch wenn die Hauptergebnisse für diese Wirkstoffe negativ ausfielen, sind diese Therapien bekanntermaßen sicher, und ihre Anwendung bei T1D im Frühstadium ist wissenschaftlich begründet. Die Global Platform for the Prevention of Autoimmune Diabetes (Globale Plattform für die Prävention von Diabetes als Autoimmunerkrankung) ist ein Gemeinschaftsprojekt mehrerer europäischer Zentren zur Erarbeitung eines Rahmens für Studien zur primären Prävention von T1D wie beispielsweise POInT (Primary Oral Insulin Trial) $[22,36]$. Im Rahmen der POInT-Studie wird untersucht, ob oral verabreichtes Insulin in der Lage ist, bei Kleinkindern mit hohem genetischen Risiko die Entwicklung von $\mathrm{Au}-$ toantikörpern und T1D hinauszuzögern oder gar zu verhindern. Eine weitere derzeit laufende Präventionsstudie ist die TrialNet Hydroxychloroquine Prevention Study (TrialNet-Studie zur Prävention mit Hydroxychloroquin), in der untersucht wird, inwieweit Hydroxychloroquin in der Lage ist, bei Probanden mit multiplen Autoantikörpern und normaler Glukosetoleranz eine Progression zu einer gestörten Glukosetoleranz oder zu klinisch manifestem T1D (oder beidem) hinauszuzögern oder gar zu verhindern. Hydroxychloroquin ist ein gut verträglicher und kostengünstiger Wirkstoff, der in der Vergangenheit zur Behandlung von Malaria eingesetzt wurde und derzeit zur Behandlung von rheumatoider Arthritis und Lupus anwendet wird. Auch wenn der Wirkstoff bislang bei T1D keine Anwendung fand, besitzt er verschiedene immunologische Eigenschaften, von denen man annimmt, dass sie günstig auf präklinischen T1D wirken, nämlich in Form einer verminderten Zellaktivierung, Antigenpräsentation und Autoantikörperproduktion [37]. Eine weitere, in der Entwicklung befindliche TrialNet-Präventionsstudie widmet sich der Untersuchung von Methyldopa, einem Alphablocker, der klinisch zur Behandlung von Bluthochdruck in der Schwangerschaft angewendet wird. Interessanterweise besitzt der Wirkstoff eine kristalline Struktur, die in den Spalt des Haupthistokompatibilitätskomplex (MHC)-Klasse-II-Moleküls HLA-DQ8 passt, welches an Insulinpeptide bindet. Insulin ist eine Zielstruktur für ein frühes Immun-Targeting bei T1D. Darüber hinaus geht man von einem Zusammenhang zwischen einem positiven InsulinAutoantikörper-Befund und einem positiven HLA DQ8-Befund aus $[38,39]$. In einer kleinen Studie zu HLA-DQ8-positiven Patienten mit neu aufgetretenem T1D blockierte Methyldopa nachweislich spezifisch die Bindung des Insulinpeptids an HLA-DQ8 und verminderte die Entzündungsantwort von T-Zellen gegenüber Insulin [40]. TrialNet strebt die Lancierung einer ähnlichen Studie an, in deren Rahmen die Wirkungen von Methyldopa auf die Antigenpräsentation bei einer DQ8-positiven Risikopopulation von Kindern und Erwachsenen mit Insulin-Antikörpern untersucht werden sollen [41].

\section{Personalisierung der Immuntherapie bei Typ-1-Diabetes}

Wie in Studien bei neu aufgetretenem T1D nachgewiesen, kann bei einigen Patienten nach der Diagnosestellung die Insulinsekretion durch eine Immuntherapie aufrecht erhalten werden. Bei einem erheblichen Anteil der Patienten unter Pharmakotherapie zeigt sich allerdings eine Verringerung der C-Peptid-Konzentration, die in etwa derjenigen bei Patienten unter Placebo entspricht. Dies ließ sich in der Teplizumab-Studie bei neu aufgetretenem T1D des Immune Tolerance Network (AbATE) beobachten, in der bei 45\% der mit Teplizumab behandelten Patienten die C-PeptidKonzentration nach 2 Jahren erhalten blieb. Bei 55\% der Patienten unter Teplizumab zeigten sich jedoch keinerlei Unterschiede im Vergleich mit den Kontrollen unter Placebo [42]. Weitere Untersuchungen sind erforderlich, um eine Therapie-Response vorhersagen und kontrollieren zu können sowie um zu entscheiden, wer wann behandelt werden sollte. Im folgenden Abschnitt werden einige erste Beobachtungen beschrieben.

\section{Kinder sprechen gut auf eine Therapie an}

Auch wenn man bei T1D mittlerweile nicht mehr von «juvenilem» Diabetes spricht, erhält eine Vielzahl von Betroffenen die Diagnose im Kindesalter und lebt mit der Erkrankung über Jahrzehnte. Mehrere Studien zu neu aufgetretenem T1D legen nahe,
58

Kompass Autoimmun 2021;3:55-61 DOI: $10.1159 / 000516244$ 
dass der Nutzen einer Immuntherapie bei Kindern leichter nachweisbar ist als bei Erwachsenen. So sind beispielsweise die positiven Ergebnisse der Studien mit Rituximab und Abatacept bei neu aufgetretenem T1D in erster Linie auf die bei Kindern beobachtete Response zurückzuführen [31, 32, 43]. Da diese Studien nicht für einen Vergleich der Wirkungen bei Erwachsenen und Kindern ausgelegt waren, bedeutet dies nicht, dass die Therapie bei Erwachsenen keine Wirkung zeigt. Tatsächlich wurde das Alter in der Teplizumab Prevention Studie nicht als Faktor im Hinblick auf ein Ansprechen ermittelt. Man weiß jedoch, dass es bei Kindern nach der Diagnose schneller zu einem absoluten Insulinmangel kommt als bei Erwachsenen [44], was auf eine aggressivere Immunaktivität bei Kindern hindeutet. De facto ist bei 35\% der Patienten, bei denen T1D im Alter von über 18 Jahren diagnostiziert wird, C-Peptid 10 bis 19 Jahre nach der Diagnose nachweisbar, im Vergleich zu lediglich bei 9\% der Patienten, bei denen T1D unter 18 Jahren diagnostiziert wird [45]. Weitere Belege liefern Insulitisprofile histopathologischer Proben [46] sowie RNA-Sequenzdaten [47]. Sie stützen die These, dass sich das Alter auf den Immunphänotyp bei T1D auswirkt. Insgesamt legen diese Beobachtungen nahe, dass - auch wenn sowohl Kinder als auch Erwachsene von frühzeitigen Maßnahmen profitieren - der Nutzen für Kinder unter Umständen deutlicher ausfällt. Darüber hinaus ist im Kindesalter das Hinauszögern einer klinischen Diagnose um mehrere Jahre klinisch von Bedeutung, da Erwachsene meist besser mit den Anforderungen eines T1D zurechtkommen als Jugendliche oder Kinder. Da die Inzidenz von T1D möglicherweise mit dem Alter abnimmt, könnte eine «Pause» in der Immunaktivität unter Umständen eine natürliche Toleranzentwicklung ermöglichen.

\section{Genetische Merkmale}

HLA-Gene tragen am stärksten zum Risiko für T1D bei, wobei jedoch auch andere Gene für Proteine mit direkten oder indirekten Immunwirkungen auf die Pathophysiologie des T1D kodieren. Interessanterweise scheinen HLA-Gene hauptsächlich zum Risiko für die Entwicklung von Autoantikörpern beizutragen und eine weniger wichtige Rolle für die Progression zu spielen, sobald multiple Antikörper vorhanden sind [48-50]. Auch wenn die Daten zur Beurteilung der Rolle von Nicht-HLA-Genen weit weniger belastbar sind, weisen einige Belege darauf hin, dass Nicht-HLA-Gene möglicherweise zur Progression von einem positiven Antikörperstatus hin zu einem manifesten T1D beitragen könnten [51]. Zudem gibt es Hinweise darauf, dass HLA-Gene das Therapieansprechen beeinflussen könnten. In der TrialNet Teplizumab Prevention Studie etwa wurde die T1D-Diagnose bei mit Teplizumab behandelten Teilnehmern mit dem Status HLA-DR3 -/DR4 ${ }^{+}$zu einem späteren Zeitpunkt gestellt, während die Krankheitsprogression bei DR3positiven/DR4-negativen Patienten mit derjenigen von Patienten unter Placebo vergleichbar war.

\section{Immuneigenschaften}

Auch wenn bislang kein Zusammenhang zwischen den immunologischen Ausgangsmerkmalen und der Therapieresponse ermit- telt wurde, lassen sich nach der Behandlung Immunphänotypen häufiger bei Respondern als bei Nicht-Respondern nachweisen. Dies ließ sich sowohl in der AbATE- als auch in der TrialNet Teplizumab Prevention Studie beobachten: Probanden mit Markern für eine T-Zell-Depletion (im Zusammenhang mit einer länger anhaltenden Antigenstimulation und einem Funktionsverlust der CD8-Effektorzellen) sprachen nachweislich besser auf eine Behandlung mit Teplizumab an [3,33]. Darüber hinaus gibt es Hinweise darauf, dass die größte Wirksamkeit einer Immuntherapie in Phasen starker Immunaktivität erzielt werden könnte, wie etwa die Teplizumab Prevention Studie nahe legt. Teilnehmer, die zu Studienbeginn eine C-Peptid-Konzentration (ein möglicher Marker für eine höhere Krankheitsaktivität) unterhalb des Medianwerts aufwiesen, sprachen besser auf die TeplizumabTherapie an als Studienteilnehmer mit einer C-Peptid-Konzentration oberhalb des Medianwerts [3]. Solche Marker könnten herangezogen werden, um therapeutische Fenster für die Initialbehandlung sowie die Behandlung während eines Rezidivs zu ermitteln.

\section{Zukunftsperspektive}

Ungeachtet bedeutender Fortschritte in den vergangenen 100 Jahren werden die Behandlungsziele für die meisten Patienten mit T1D nicht erreicht, und bei ihnen besteht weiterhin das Risiko für Komplikationen. Dies legt nahe, dass die aktuellen Insulinabgabesysteme allein nicht ausreichen. Das Krankheitsmodell bei T1D hat sich verändert: Eine Insel-Autoimmunität, bestimmt durch 2 oder mehr T1D-spezifische Autoantikörper, gilt mittlerweile als das erste Stadium des T1D. Eine frühe immuntherapeutische Behandlung zur Vermeidung von Gewebezerstörung und Funktionsverlusten stellt bei anderen Autoimmunkrankheiten wie rheumatoider Arthritis oder Multipler Sklerose bereits den Versorgungsstandard dar. Wahrscheinlich wird eine Immuntherapie vor und/oder kurz nach der klinischen Diagnose auch bei T1D Teil der Routineversorgung werden. Auch wenn in der vorliegenden Übersichtsarbeit nicht darauf eingegangen wird, könnten auch Ansätze zum direkten Targeting von Betazellen Bestandteil der T1D-Therapie werden.

Erwägung alternativer Studiendesigns für eine beschleunigte Aufnahme in und Durchführung von Studien bei gleichzeitiger Kostensenkung

Eine Analyse von Surrogat-Endpunkten zu einem früheren Zeitpunkt in der Entwicklung von T1D könnte den für eine Bewertung der Behandlungswirksamkeit erforderlichen Zeitaufwand reduzieren. In Anbetracht des unausweichlichen Auftretens eines manifesten T1D bei Vorliegen von 2 oder mehr Autoantikörpern könnte die Entwicklung einer Insel-Autoimmunität als intermediärer Endpunkt für Studien zur Primärprävention dienen. Da sich aus einer Insel-Autoimmunität mit normaler Glukosetoleranz eine Insel-Autoimmunität mit gestörter Glukosetoleranz und nachfolgend ein klinischer T1D entwickelt, wird gleichermaßen das Auftreten einer gestörten Glukosetoleranz als Studien- 
endpunkt in Studien zur Überprüfung von Therapien auf ihre Fähigkeit, die Progression von einer Insel-Autoimmunität zu T1D hinauszuzögern, herangezogen. Dieser Ansatz wird, wie oben beschrieben, im Rahmen der TrialNet-Studien «Abatacept in Prevention» (Abatacept zur Prävention) und «Hydroxychloroquine in Prevention» (Hydroxychloroquin zur Prävention) untersucht [16, 37]. Adaptive Studiendesigns [52], Studien mit mechanistischen oder kombinierten Endpunkten [53] oder einarmige Studien könnten alternativ zum «Goldstandard» der placebokontrollierten Studie dienen, sowohl im Hinblick auf eine vereinfachte Rekrutierung von Probanden als auch auf eine Kostensenkung. In Anbetracht des umfassenden Datenkorpus zur Pathophysiologie des T1D ist es machbar, im Rahmen einer einarmigen Studie anhand bereits vorhandener Datensätze die Notwendigkeit einer Placebo-Kohorte abzuklären.

Ein Screening der Allgemeinbevölkerung wird erforderlich sein, um die Mehrzahl der Personen mit Insel-Autoimmunität zu identifizieren

Wie oben beschrieben könnte dies durch einen Gentest bei der Geburt und anschließende regelmäßige Untersuchungen auf Autoantikörper bei Kindern bewerkstelligt werden, die eine Hochrisiko-Genkonfiguration oder T1D in der Familienanamnese aufweisen, oder aber durch regelmäßige Tests auf Autoantikörper der Bevölkerung insgesamt. Wie auch immer die Vorgehensweise aussieht, ein Screening der Allgemeinbevölkerung ist kostenintensiv und wird erhebliche Anstrengungen in Form einer Aufklärung und Beratung derjenigen Personen erfordern, bei denen ein Risiko festgestellt wird und die keine Erfahrung mit T1D haben. Die Untersuchung von Familienangehörigen auf ein Risiko für T1D kann Ängste schüren. Zu erfahren, dass bei sich selbst oder dem eigenen Kind ein hohes Risiko besteht, ist belastend und schafft Unsicherheit. Diskriminierung oder eine Änderung der Lebensplanung können mögliche Folgen sein. Ungeachtet dessen lassen Studien darauf schließen, dass sich die anfänglichen Ängste im Zusammenhang mit einer solchen Untersuchung mit wiederholten Tests allmählich verflüchtigen [54]. Eine T1D-Diagnose im Rahmen eines Risiko-Screenings - so haben weitere Arbeiten ergeben - ist mit geringeren Belastungen verbunden als wenn Familien die Diagnose ohne ein solches Screening erhalten [55]. Die Frlda-Studie lässt ein vergleichbares Muster bei Menschen ohne T1D in der Familie vermuten: Der elterliche Stress war nach Diagnostizierung eines T1D im Frühstadium höher als bei Eltern mit negativem Testergebnis, aber das Stressniveau sank im Jahr nach dem Test wieder [9]. Wie in der Frlda-Studie berichtet, war der elterliche Stress zudem in der Kohorte derjenigen, bei deren Kindern ein asymptomatischer T1D im Frühstadium diagnostiziert wurde, niedriger als in einer Kohorte von Eltern aus der DiMelli-Studie [56], bei deren Kindern ein klinisch manifester T1D ohne vorheriges Staging diagnostiziert wurde.

Eine dauerhafte Immuntherapie könnte notwendig sein Die anfänglichen Hoffnung, eine kurzfristige Immuntherapie würde durch Wiederherstellung der Selbsttoleranz zu einer lang- fristigen oder nachhaltigen Remission führen, hat sich nicht bewahrheitet. Dies gilt auch für alle anderen Autoimmunkrankheiten. Wahrscheinlich wird eine andauernde oder intermittierende Behandlung erforderlich sein, um die Funktion der Betazellen zu erhalten und einer Krankheitsprogression vorzubeugen [57]. Diese Strategie wurde bislang bei T1D nicht untersucht, wird jedoch mit Einführung neuer und sicherer Immuntherapien zur Langzeitbehandlung anderer Autoimmunkrankheiten zunehmend praktikabel.

Mögliche Synergieeffekte durch eine kombinierte oder sequenzielle Anwendung von Therapien

Kombinierte Immuntherapien werden bereits bei anderen immunvermittelten Erkrankungen angewendet, etwa die Kombination von Methotrexat und Etanercept bei rheumatoider Arthritis [58] oder von Azathioprin plus Infliximab bei Morbus Crohn [59]. TrialNet plant die Durchführung einer Studie bei einer Hochrisikopopulation mit multiplen Autoantikörpern und gestörter Glukosetoleranz zur Beurteilung der Wirkung von Rituximab mit anschließender Gabe von Abatacept auf die Krankheitsprogression. Das Konzept der Kombinationsbehandlung ergab sich aus den Ergebnissen der jeweiligen Monotherapie-Studien mit Abatacept bzw. Rituximab bei neu aufgetretenem T1D. In der Abatacept-Studie war bei Teilnehmern mit einer hohen B-Zell-Signatur sechs Monate nach Behandlungsbeginn die Wahrscheinlichkeit eines Ansprechens auf die Abatacept-Therapie niedriger [60]. Entsprechend war in der Rituximab-Studie bei Teilnehmern mit einer hohen aktivierten T-Zell-Signatur sechs Monate nach der Behandlung die Wahrscheinlichkeit eines Ansprechens auf die Rituximab-Therapie ebenfalls niedriger [61]. In der geplanten TrialNet-Studie zu der Kombinationsbehandlung werden vier wöchentliche Behandlungen mit Rituximab zu einer Depletion der $\mathrm{CD} 20^{+}$-B-Zellen führen. Vier Monate später, vor einer Erholung der B-Zellen, beginnt dann die 2-jährige Behandlung mit Abatacept. Man geht davon aus, dass Abatacept die für die Regeneration der B-Zellen notwendige Unterstützung durch die T-Zellen stört und so die Wiederherstellung autoreaktiver B-Zellen verhindert.

\section{Niedrigere Kosten und höhere Anwendungsfreundlichkeit der Immuntherapie zu erwarten}

Zwar ist eine Immuntherapie kostenintensiv und umständlich, dies gilt jedoch ebenso für ein ganzes Leben mit T1D. Laut Schätzung im Bericht des US-amerikanischen Health Care Cost Institute von 2019 (www.healthcostinstitute.org/research/publications/ entry/spending-on-individuals-with-type-1-diabetes-and-the-role-of-rapidly-increasing-insulin-prices) beliefen sich die durchschnittlichen Gesundheitsausgaben im Jahr 2016 für eine Person mit T1D auf 18494 US-Dollar, indirekte Kosten nicht mitgerechnet. Auch wenn die konkreten Kosten für die jeweiligen Behandlungsansätze derzeit noch unklar sind, ist zu erwarten, dass Immuntherapien in oraler und subkutaner Form, die zuhause verabreicht werden können, die Praktikabilität einer Immuntherapie bei T1D erhöhen und dass die zunehmende Verfügbarkeit «bioäquivalenter» oder generischer Präparate zu Kostensenkungen
60

Kompass Autoimmun 2021;3:55-61

DOI: $10.1159 / 000516244$ 
führen werden. Somit erreichen wir einen Punkt, an dem selbst eine präventive Langzeit-Immuntherapie unter Umständen kosteneffektiver sein könnte als eine lebenslange Behandlung von T1D.

\section{Mehr Überzeugungsarbeit ist erforderlich}

Um den Paradigmenwechsel bezüglich der Behandlung von T1D einzuleiten, ist eine vielfältige Herangehensweise notwendig: Es gilt, die trotz Behandlungserfolgen fortbestehende Belastung durch T1D zu verstehen, das neue Krankheitsmodell von T1D, nämlich dass T1D lange vor Auftreten einer Hyperglykämie beginnt, zur Norm zu erklären und das Potenzial der Immuntherapie, die Grunderkrankung zu modifizieren, anzuerkennen. Nicht von T1D Betroffene (einschließlich der Ärzteschaft) könnten den Eindruck haben, T1D lasse sich mit minimalem Aufwand und minimalem Risiko behandeln. Dabei wird den Nachweisen dafür, dass die Mehrzahl der Menschen mit T1D die empfohlenen Behandlungsziele nicht erreicht, keinerlei Rechnung getragen. Infolge dessen entschuldigen sich Menschen mit T1D (und Typ2-Diabetes) womöglich dafür, dass sie ihre Erkrankung nur unzureichend in den Griff bekommen, anstatt anderen gegenüber ihren täglichen Kampf mit der Krankheit, einschließlich der Kosten und der mentalen/emotionalen Belastung, zuzugeben. Neben der nicht in Aussicht stehenden Abhilfe in Bezug auf die Hyperglykämie ist eine stärkere Sensibilisierung dafür, dass eine InselAutoimmunität die Vorstufe eines klinisch manifesten T1D dar- stellt, notwendig. Vor diesem Hintergrund scheint ein Paradigmenwechsel weg von der Behandlung der Hyperglykämie hin zu einer Prävention der Hyperglykämie nur selbstverständlich. Auch wenn die Immuntherapie für eine klinische Anwendung bei T1D noch nicht ausgereift ist, erscheint diese Möglichkeit in Anbetracht der Ergebnisse der Teplizumab Prevention Studie - der ersten Studie, die den Nachweis erbringt, dass eine klinische T1DDiagnose durch eine Immuntherapie hinausgezögert werden kann - realistischer als jemals zuvor.

\section{Erklärung zur Finanzierung}

Die vorliegende Arbeit wurde durch Zuwendungen der US-amerikanischen National Institutes of Health mit den Nummern U01DK106693, U01USF6163-1082-00-BY und U01DK103282 finanziell unterstützt.

Die Geldgeber hatten keinerlei Einfluss auf Studienaufbau, Datenerhebung und -auswertung, die Entscheidung zur Veröffentlichung oder die Erstellung des Manuskripts.

\section{Lizenzangabe}

Lord S, Greenbaum CJ. Insulin is necessary but not sufficient: changing the therapeutic paradigm in type 1 diabetes. F1000Res. 2020; 9:F1000 Faculty Rev-827 (DOI: 10.12688/f1000research.21801.1). ${ }^{\circ} 2020$ Lord S and Greenbaum CJ (Übersetzung; Abbildung 2 und Notes gekürzt), lizensiert unter CC BY 4.0 (https://creativecommons.org/licenses/by/4.0/deed.de).

\section{Literatur}

Die Literatur ist unter www.karger.com/doi/10.1159/000516244 abrufbar. 